\title{
The Oxygen K edge in Strontium Titanate and Lanthanum Titanate
}

\author{
M.P. Kocher**, D.A. Muller* and P. Rez** \\ Lucent Technologies, 7500 Mountain Avenue, Murray Hill, NJ 07974 \\ Department of Physics and Astronomy, Arizona State University, Tempe, AZ 85287-1504
}

Recently there has been considerable interest in titanates for applications in electronic components such as varistors, and as candidates for high dielectric constant materials to replace silicon dioxide in MOS transistors. Superlattices composed of strontium and lanthanum titanate layers should show one dimensional charge modulation due to the formal change in valence from $4+$ in $\mathrm{SrTiO}_{3}$ to $3+$ in $\mathrm{LaTiO}_{3}$. Multilayers grown by pulsed laser deposition have been examined by a combination of high angle annular dark field imaging and electron energy loss spectrometry [1]. The titanium $\mathrm{L}_{23}$ edges the lanthanum $\mathrm{M}_{45}$ edge and the oxygen $\mathrm{K}$ edge were all recorded with an energy resolution of about $0.9 \mathrm{eV}$ and a spatial resolution of about $1.9 \AA$. The titanium $\mathrm{L}_{23}$ near edge structure can be decomposed into a linear combination of the spectra from $\mathrm{Ti}^{3+}$ and $\mathrm{Ti}^{4+}$ and can be well modeled using atomic multiplet theory [2]. This is because the octahedral symmetry at the Ti site is preserved, any change in valence is accommodated at the Ti site, and the Ti $2 p-3 d-3 d$ couplings in the presence of a core hole are large compared with the width of the $3 \mathrm{~d}$ bands.

The oxygen $\mathrm{K}$ edges from the lanthanum and strontium compounds also show different near edge structures as shown in Fig. 1. It is usually the case with oxides that core hole effects are negligible and that the near edge structure is well represented by the p projected density of states at the oxygen site [3]. Standard electronic structure codes giving projected densities of states can therefore be used. Densities of states at the atomic sites for both strontium and lanthanum titanate were calculated from the band structure and the projection operator output of the VASP code [4]. A Gaussian smearing technique was used to calculate the projected densities of states from a grid of $5 \times 5 \times 5 \mathrm{k}$ points. It was found that the results were negligibly different from calculations using the tetrahedron method. The calculated $\mathrm{p}$ densities of states in Fig 2 show the same features as the experimental spectra. The threshold peak is lower in the lanthanum compound and peak B is at about $532 \mathrm{eV}$ as compared to $535 \mathrm{eV}$ from the strontium compound. Peak C, almost certainly an EXAF peak, is at $542 \mathrm{eV}$ in both compounds, which is not surprising given that the lattice parameters are almost identical. Comparing the oxygen $\mathrm{p}$ density of states with the lanthanum and strontium d densities of states given in Fig 3, shows that peak B is a result of a transition to a state derived from $\mathrm{O} p$ and $\mathrm{La}$ or $\mathrm{Sr} \mathrm{d}$ orbitals. Calculations modeling a core hole using the $\mathrm{Z}+1$ approximation at the center of a 32-atom supercell gave $p$ densities of states that were in poor agreement with experiment, especially in the threshold region where the relative intensity of peak A was grossly overestimated. This is consistent with previous findings that ground state calculations, when done correctly, are better for oxygen K edges.

\section{References}

[1] A. Ohtomo, D.A. Muller, J.L. Grazul and H.Y. Hwang, Nature 419 (2002) 478.

[2] A. Fujimori et al, Phys. Rev. B 46 (1992) 9841.

[3] M. Grioni et al ,Phys. Rev. B 49, (1989) 4886.

[4] G. Kresse and J. Furthmuller Phys. Rev. B 54, (1996) 11169.

[5] PR would like to acknowledge support from NSF grant DMR 99-72670 


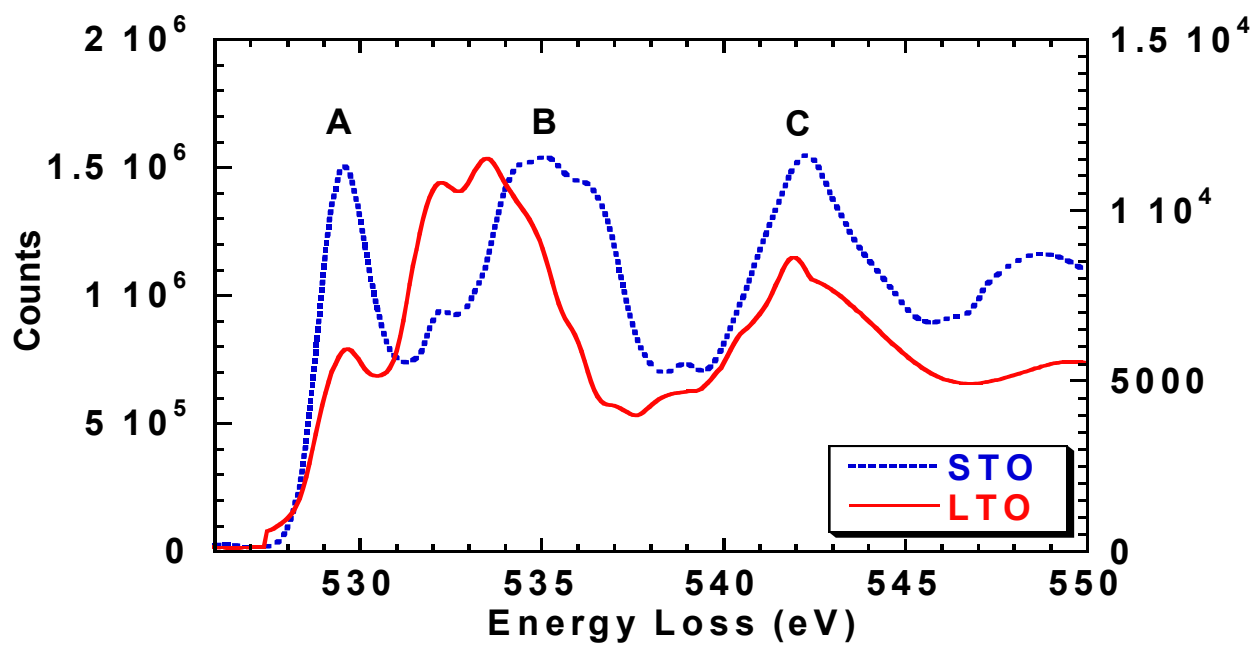

FIG 1 Experimental O K edges from $\mathrm{SrTiO}_{3}$ (dashed line) $\mathrm{LaTiO}_{3}$ (solid line).

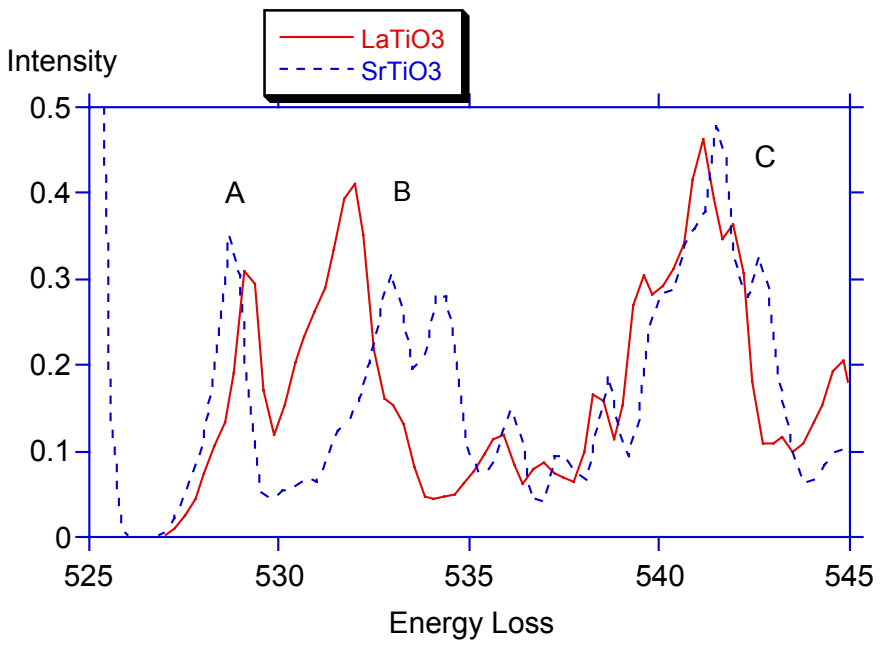

FIG 2. Calculated O p density of states for from $\mathrm{SrTiO}_{3}$ (dashed line) $\mathrm{LaTiO}_{3}$ (solid line)

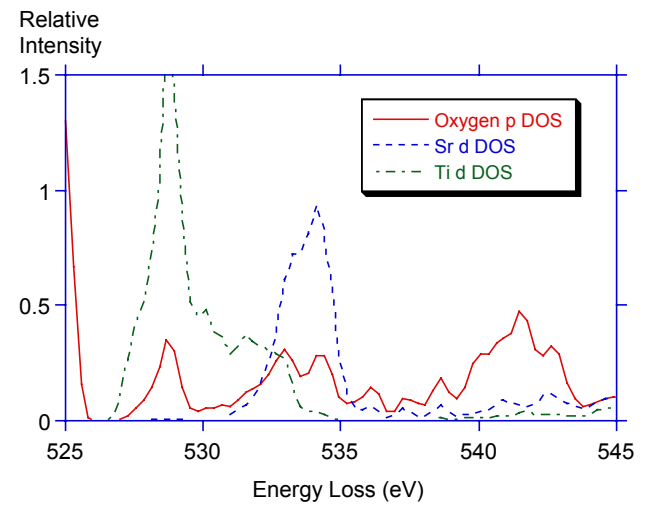

FIG 3(a).

$\mathrm{SrTiO}_{3} \mathrm{O}$ p DOS, Sr d DOS, Ti d DOS

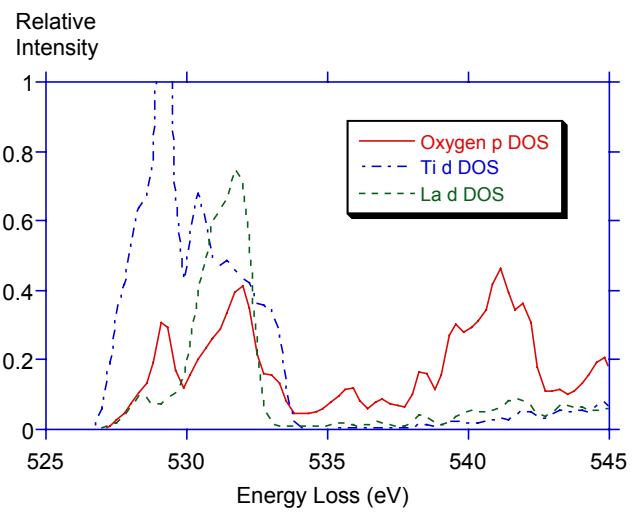

FIG 3(b).

$\mathrm{LaTiO}_{3}$ O p DOS, La d DOS, Ti d DOS 